\title{
Computing the numerical range of Krein space operators
}

\author{
N. Bebiano, J. da Providência, A. Nata ${ }^{\ddagger}$, and J.P. da Providência§
}

December 31, 2013

\begin{abstract}
Consider the Hilbert space $(\mathcal{H},\langle\cdot, \cdot\rangle)$ equipped with the indefinite inner product $[u, v]=$ $v^{*} J u, u, v \in \mathcal{H}$, where $J$ is an indefinite self-adjoint involution acting on $\mathcal{H}$. The Krein space numerical range $W_{J}(T)$ of an operator $T$ acting on $\mathcal{H}$ is the set of all the values attained by the quadratic form $[T u, u]$, with $u \in \mathcal{H}$ satisfying $[u, u]= \pm 1$. We develop, implement and test an alternative algorithm to compute $W_{J}(T)$ in the finite dimensional case, constructing 2 by 2 matrix compressions of $T$ and their easily determined ellipses and hyperbolical numerical ranges. The numerical results reported here indicate that this method is very efficient, since it is faster and more accurate than either of the existing algorithms. Further, it may yield easy solutions for the inverse indefinite numerical range problem. Our algorithm uses an idea of Marcus and Pesce from 1987 for generating Hilbert spaces numerical ranges of matrices of size $n$.
\end{abstract}

AMS subject classification: 15A60; 15A63

Key words: Indefinite inner product; Krein space; numerical range; compression

\section{Introduction}

Let $J$ be an indefinite self-adjoint involution acting on a Hilbert space $(\mathcal{H},\langle\cdot, \cdot\rangle)$. Define the sesquilinear form (indefinite inner product) associated with $J$ by $[u, v]=\langle J u, v\rangle=v^{*} J u, u, v \in \mathcal{H}$. The indefinite numerical range of a linear operator $T: \mathcal{H} \rightarrow \mathcal{H}$ is the set of complex numbers

$$
W_{J}(T)=\left\{\frac{[T w, w]}{[w, w]}: w \in \mathcal{H},[w, w] \neq 0\right\} .
$$

This concept generalizes the well-known (classical) numerical range, defined by

$$
W(T)=\left\{\frac{\langle T w, w\rangle}{\langle w, w\rangle}: w \in \mathcal{H},\langle w, w\rangle \neq 0\right\} .
$$

${ }^{*}$ CMUC, University of Coimbra, Department of Mathematics, P 3001-454 Coimbra, Portugal (bebiano@mat.uc.pt)

${ }^{\dagger}$ University of Coimbra, Department of Physics, P 3004-516 Coimbra, Portugal (providencia@teor.fis.uc.pt)

${ }^{\ddagger}$ CMUC, Polytechnic Institute of Tomar, Department of Mathematics, P 2300-313 Tomar, Portugal (anata@ipt.pt)

${ }^{\S}$ Depatamento de Física, Univ. of Beira Interior, P-6201-001 Covilhã, Portugal (joaodaprovidencia@daad-alumni.de) 
The numerical range is a useful tool in the study of matrices and operators, that has been investigated extensively (e.g., see [1, 8] and [17] and references therein). Several results are known which connect analytic and algebraic properties of an operator with the geometrical properties of its numerical range. Likewise, the indefinite numerical range motivated the interest of researchers (see $[2,6,13,14$, 15]), which in particular have investigated these relations in the Krein space setting. The indefinite numerical range, although sharing some analogous properties with the classical numerical range, has a quite different behavior. In contrast with the classical case, $W_{J}(T)$ is generally neither closed nor bounded [15, Section 2]. On the other hand, $W_{J}(T)$ may not be convex [15].

We also define the related sets

$$
W_{J}^{+}(T)=\left\{\frac{[T w, w]}{[w, w]}: w \in \mathcal{H},[w, w]>0\right\}
$$

and

$$
W_{J}^{-}(T)=\left\{\frac{[T w, w]}{[w, w]}: w \in \mathcal{H},[w, w]<0\right\} .
$$

It is easy to check that $W_{-J}^{+}(T)=-W_{J}^{-}(T)$ and

$$
W_{J}(T)=W_{J}^{+}(T) \cup W_{J}^{-}(T) .
$$

Thus, we can focus our study on $W_{J}^{+}(T)$ and translate the results on $W_{J}^{+}(T)$ to $W_{J}^{-}(T)$ and $W_{J}(T)$.

We mostly consider $\mathcal{H}=\mathbb{C}^{n}$ and we denote by $M_{n}$ the algebra of $n \times n$ complex matrices. We assume that the inertia of $J$ is $(r, n-r)$, i.e., $J$ has $r$ positive and $n-r$ negative eigenvalues. According to Sylvester law of inertia [11, p.222-223], there exists a non-singular matrix $S \in M_{n}$ such that $S^{*} J S=I_{r} \oplus-I_{n-r}$. Clearly, $W_{I_{r} \oplus-I_{n-r}}\left(S^{-1} T S\right)=W_{J}(T)$. So, without loss of generality we shall consider $J=I_{r} \oplus-I_{n-r}$.

In this paper we revisit the question of numerically determining $W_{J}(T)$, which has already already deserved the attention of researchers (cf. [4] and [14]). Nevertheless, the existing methods are not efficient in some cases, namely, because this set is very often unbounded and so it is difficult to approach accurately its boundary. Our main aim is to present an alternative algorithm for plotting $W_{J}(T)$, which refines an idea used by Marcus and Pesce to numerically determine the classical numerical range [16] (see also [18]). As it will be shown, our method has clear advantages over the existing ones [4, 13], both in accuracy and in execution time (cf. Section 5). Further, the presented algorithm is crucial for the line of attack we adopt for solving the inverse indefinite numerical range stated as follows: for a given point $z \in W_{J}(T)$, determine a vector $u \in \mathbb{C}^{n}$ such that $z=[T u, u] /[u, u]$. For more details see [7]

This paper is organized as follows. In Section 2, results used throughout our investigation are surveyed. In Section 3, the indefinite numerical range is described as a union of elliptical and hyperbolical disks. In Section 4, an algorithm to plot $W_{J}(T)$ based on the previous result is presented. In 
Section 5, numerical examples illustrating the proposed approach are provided, and the performance of the different algorithms is discussed. We end with some conclusions in Section 6. The images were computed numerically using MATLAB.

\section{Prerequisites}

We start recalling some useful facts. A matrix $T \in M_{n}$ is called $J$-Hermitian (or $J$-self-adjoint), if $T=T^{\#}$, where $T^{\#}=J T^{*} T$ is the $J$-adjoint of $T$. Any matrix $T$ may be uniquely written in the form $T=\operatorname{Re}^{J} T+i \operatorname{Im}^{J} T$, where $\operatorname{Re}^{J} T:=1 / 2\left(T+T^{\#}\right)$ and $i \operatorname{Im}^{J} T:=1 / 2\left(T-T^{\#}\right)$ are $J$-Hermitian matrices. The spectrum of a $J$-Hermitian matrix is symmetric relatively to real axis. It is well-known that $W_{J}(T) \subseteq \mathbb{R}$ if and only if $T$ is $J$-self-adjoint. We clearly have $W_{J}\left(\operatorname{Re}^{J} T\right)=\operatorname{Re}\left(W_{J}(T)\right) \subseteq \mathbb{R}$ and $W_{J}\left(\operatorname{Im}^{J} T\right)=\operatorname{Im}\left(W_{J}(T)\right) \subseteq \mathbb{R}$. Further, if $T$ has complex eigenvalues, then $W_{J}(T)$ is the whole real line [2].

A matrix $U$ is $J$-unitary if $U U^{\#}=I$. Assume that $T$ is a $J$-Hermitian matrix with real spectrum and $J$-unitarily diagonalizable. Let define

$$
\sigma_{J}^{ \pm}(T)=\left\{\lambda \in \mathbb{R}: \exists x \in \mathbb{C}^{n}, \quad[x, x]= \pm 1, \quad T x=\lambda x\right\}
$$

Throughout, we shall be specially concerned with the class of matrices $T \in M_{n}$, for which there exists $\theta \in\left[\theta_{1}, \theta_{2}\right]$, with $0<\theta_{2}-\theta_{1}<\pi$, such that the $J$-Hermitian matrix

$$
H_{\theta}:=\operatorname{Re}^{J}\left(\mathrm{e}^{-i \theta} T\right)=\frac{1}{2}\left(\mathrm{e}^{-i \theta} T+\mathrm{e}^{i \theta} T^{\#}\right),
$$

has real eigenvalues satisfying the following conditions:

(i) $\lambda_{1}\left(H_{\theta}\right) \geq \cdots \geq \lambda_{r}\left(H_{\theta}\right) \in \sigma_{J}^{+}\left(H_{\theta}\right)$;

(ii) $\lambda_{r+1}\left(H_{\theta}\right) \geq \cdots \geq \lambda_{n}\left(H_{\theta}\right) \in \sigma_{J}^{-}\left(H_{\theta}\right)$;

(iii) $\lambda_{r}\left(H_{\theta}\right)>\lambda_{r+1}\left(H_{\theta}\right)$.

For $T$ in this class, $W_{J}(T)$ is non-degenerate, i.e., is not a singleton, a whole line (possibly without a point), the whole complex plane (possibly without a line) or the union of two non-intersecting half planes. This class of matrices will be denoted by $\mathcal{N D}$, the acronym for non-degenerate.

In our subsequent discussion, we shall use the following basic properties. We have $W_{J}(T)=\{\lambda\}$ if and only if $T=\lambda I$, and for any $T$ and $\alpha, \beta \in \mathbb{C}, W_{J}(\alpha T+\beta I)=\alpha W_{J}(T)+\beta$. The $J$-unitary transformations preserve the shape of Krein space numerical ranges, $W_{J}\left(U^{\#} T U\right)=W_{J}(T)$. The set $W_{J}(T)$ is pseudo-convex [15], that is, for any pair of distinct points $x, y$ either the line segment with 
end points $x, y$ is contained in $W_{J}(T)$, or the two half-lines $(1-t) x+t y$ for $t \leq 0$ or $t \geq 1$ are there contained.

We denote the boundary of $W_{J}(T)$ by $\partial W_{J}(T)$. The supporting lines of $W_{J}(T)$ are the supporting lines of the convex sets $W_{J}^{+}(T)$ and $W_{J}^{-}(T)$. If $\ell$ is a supporting line of $W_{J}(T)$ and $\ell \cap \partial W_{J}(T)$ contains more than one point, then $\ell \cap \partial W_{J}(T)$ is called a flat portion on the boundary of $W_{J}(T)$ [5]. There is a flat portion in $\partial W_{J}^{+}(T)$ if and only if there exists $\theta$ such that the smallest eigenvalue $\lambda_{r}\left(H_{\theta}\right)$ in $\sigma_{J}^{+}\left(H_{\theta}\right)$ is multiple and the set $\left\{z=[T u, u] /[u, u]: H_{\theta} u=\lambda_{r}\left(H_{\theta}\right) u\right\}$ is not a singleton. An analogous result is valid for $W_{J}^{-}(T)$.

A point $z \in \partial W_{J}^{+}(T)$ is called a corner of $W_{J}^{+}(T)$ if it is in more than one supporting line. In [14, Theorem 3.1], it was proved that if $z$ is a corner, then it is an eigenvalue of $T$.

\section{$3 \quad W_{J}(T)$ as a union of elliptical and hyperbolical discs}

One important result in the Krein space numerical ranges is the hyperbolical range theorem [2] asserting that for a linear operator $T \in M_{2}$, with eigenvalues $\lambda_{1}$ and $\lambda_{2}$, and a self-adjoint involution $J_{2}, W_{J_{2}}(T)$ is bounded by a (possibly degenerate) 2-component hyperbola with foci $\lambda_{1}$ and $\lambda_{2}$, and transverse and non-transverse axis of length $\sqrt{\operatorname{Tr}\left(T^{\#} T\right)-2 \operatorname{Re}\left(\lambda_{1} \bar{\lambda}_{2}\right)}$ and $\sqrt{\left|\lambda_{1}\right|^{2}+\left|\lambda_{2}\right|^{2}-\operatorname{Tr}\left(T^{\#} T\right)}$, respectively. For the classical numerical range, the elliptical range theorem [12] states that if $T \in M_{2}$, then $W(T)$ is a (possibly degenerate) closed elliptical disc, whose foci are the eigenvalues of $T, \lambda_{1}$ and $\lambda_{2}$ and the lengths of the axis are $\sqrt{\operatorname{Tr}\left(T^{*} T\right)-2 \operatorname{Re}\left(\lambda_{1} \bar{\lambda}_{2}\right)}$, and $\sqrt{\operatorname{Tr}\left(T^{*} T\right)-\left|\lambda_{1}\right|^{2}-\left|\lambda_{2}\right|^{2}}$. For $T$ acting on higher dimensional spaces, the shape of $W_{J}(T)$ is more complicated. In this section, we prove a theorem that reduces the general case to the bi-dimensional one.

Let $P \in M_{2}$ be a $J$-orthogonal projection, i.e., $P^{2}=P, P^{\#}=P$. For $T \in M_{n}$, we recall that the restriction of $P T P$ to the range of $P$ is called a 2-dimensional compression of $T$. In matrix form

$$
T_{x y}=\left[\begin{array}{cc}
\epsilon_{x}[T x, x] & \epsilon_{x}[T y, x] \\
\epsilon_{y}[T x, y] & \epsilon_{y}[T y, y]
\end{array}\right],
$$

where $x$ e $y$ are real $J$-orthonormal column $n$-tuples, i.e.,

$$
[x, y]=0, \quad \epsilon_{x}=[x, x]= \pm 1, \quad \epsilon_{y}=[y, y]= \pm 1, \quad P x=x, \quad \text { and } \quad P y=y .
$$

Explicitly, we have $P T P=T_{x y} \oplus 0_{n-2}$, the zero block of size $n-2$.

The following theorem will be applied to the problem of devising an effective procedure for generating the indefinite numerical range of an arbitrary $n \times n$ complex matrix. 
Theorem 3.1 Let $T \in M_{n}$ and $J=I_{r} \oplus-I_{n-r}$. Then $W_{J}(T)$ is the union of all the sets

$$
\left(\bigcup_{\substack{x, y \in \mathbb{R}^{n} \\[x, x]=[y, y]=1}} W_{J_{x y}}\left(T_{x y}\right)\right) \bigcup\left(\bigcup_{\substack{x, y \in \mathbb{R}^{n} \\[x, x]=[y, y]=-1}} W_{J_{x y}}\left(T_{x y}\right)\right) \bigcup\left(\bigcup_{\substack{x, y \in \mathbb{R}^{n} \\[x, x]=-[y, y]=1}} W_{J_{x y}}\left(T_{x y}\right)\right),
$$

where $T_{x y}$ is the matrix (2), $x$ and $y$ run over all pairs of real $J$-orthonormal vectors and $J_{x y}=$ $\operatorname{diag}\left(\epsilon_{x}, \epsilon_{y}\right)$, with $\epsilon_{x}$ and $\epsilon_{y}$ given by (3).

Proof. Let $w=u+i v$ be a complex vector in which $u$ and $v$ are real $n$-vectors. Assume that $[w, w] \neq 0$, and $[T w, w] /[w, w] \in W_{J}^{+}(T)$. (If $[T w, w] /[w, w] \in W_{J}^{-}(T)$ a similar treatment holds.) Thus,

$$
1=[w, w]=[u, u]+[v, v] .
$$

If $v=\alpha u, \alpha \in \mathbb{R}$, then $w=u+i \alpha u=(1+i \alpha) u$ and so

$$
[T w, w]=[T|1+i \alpha| u,|1+i \alpha| u]
$$

Since $|1+i \alpha| u$ is a real $J$-unit vector, from (5) we infer that $[T w, w] \in W_{J_{x y}}^{+}\left(T_{x y}\right)$, where $x=|1+i \alpha| u$ and $y$ is chosen to be a real $J$-unit vector such that $[x, y]=0$.

If $u$ and $v$ are linearly independent, then $[w, w]=[u, u]+[v, v] \neq 0$. Assume that $[u, u] \neq 0$, and let

$$
x=\frac{u}{\sqrt{|[u, u]|}} .
$$

Consider

$$
s=[u, v] u-[u, u] v,
$$

so that $[u, s]=0$. Assume that $[s, s]=[u, u][u, v]([u, u]-[u, v]) \neq 0$ and let

$$
y=\frac{s}{\sqrt{|[s, s]|}} .
$$

We may write

$$
w=u+i v=\alpha_{x} x+\alpha_{y} y,
$$

where

$$
\alpha_{x}=\sqrt{|[u, u]|}\left(1+i \frac{[u, v]}{[u, u]}\right), \quad \alpha_{y}=-i \frac{\sqrt{|[s, s]|}}{[u, u]} .
$$

We obtain

$$
\begin{aligned}
& {[T w, w]=\alpha_{x} \bar{\alpha}_{x}[T x, x]+\alpha_{y} \bar{\alpha}_{y}[T y, y]+\alpha_{x} \bar{\alpha}_{y}[T x, y]+\alpha_{y} \bar{\alpha}_{x}[T y, x]} \\
& =\left[\bar{\alpha}_{x}, \bar{\alpha}_{y}\right]\left[\begin{array}{cc}
\epsilon_{x} & 0 \\
0 & \epsilon_{y}
\end{array}\right]\left[\begin{array}{ll}
{[T x, x] \epsilon_{x}} & {[T y, x] \epsilon_{x}} \\
{[T x, y] \epsilon_{y}} & {[T y, y] \epsilon_{y}}
\end{array}\right]\left[\begin{array}{l}
\alpha_{x} \\
\alpha_{y}
\end{array}\right] .
\end{aligned}
$$


Further,

$$
[w, w]=\alpha_{x} \bar{\alpha}_{x}[x, x]+\alpha_{y} \bar{\alpha}_{y}[y, y]=\left[\bar{\alpha}_{x}, \bar{\alpha}_{y}\right]\left[\begin{array}{cc}
\epsilon_{x} & 0 \\
0 & \epsilon_{y}
\end{array}\right]\left[\begin{array}{l}
\alpha_{x} \\
\alpha_{y}
\end{array}\right]
$$

and we easily find

$$
\frac{[T w, w]}{[w, w]}=\frac{\left[T_{x y} z, z\right]}{[z, z]}
$$

where $z=\left[\alpha_{x}, \alpha_{y}\right]^{T}$. The equality (9) shows that any element in $W_{J}^{+}(T)$ belongs to some $W_{J_{x y}}\left(T_{x y}\right)$.

If $[s, s]=0$, we perturb $w$ so that $[s, s] \neq 0$. For this purpose, we consider $w^{\prime}=u^{\prime}+i v^{\prime}, u^{\prime}, v^{\prime} \in \mathbb{R}$, such that $\left[u^{\prime}, u^{\prime}\right]\left[u^{\prime}, v^{\prime}\right]\left(\left[u^{\prime}, u^{\prime}\right]-\left[u^{\prime}, v^{\prime}\right]\right) \neq 0$ and replace $w$ by $w_{\epsilon}=\epsilon w+(1-\epsilon) w^{\prime}$. For a sufficiently small $\epsilon$, we have

$$
\left[u_{\epsilon}, u_{\epsilon}\right]\left[u_{\epsilon}, v_{\epsilon}\right]\left(\left[u_{\epsilon}, u_{\epsilon}\right]-\left[u_{\epsilon}, v_{\epsilon}\right]\right) \neq 0 .
$$

Further, the point generated by $w_{\epsilon}$ is in the neighborhood of the point generated by $w$, and approaches it as $\epsilon \rightarrow 0$.

The reciprocal inclusion is a consequence of the following facts. Any 2-dimensional real $J$ orthogonal compression of $T$ is a 2 -square principal submatrix of a matrix $J$-orthogonally similar to $T$, and $W_{J}(T)$ is invariant under $J$-orthogonal similarities. Moreover, $W_{J^{\prime}}(B) \subset W_{J}(T)$ for any principal submatrix $B$ of $T$ and $J^{\prime}$ a conformally defined principal submatrix if $J$.

\section{Algorithms for plotting $W_{J}(T)$}

One typical method to generate $W_{J}(T)$ consists on the determination of the algebraic curve $\partial W_{J}(T)$ (cf. [17]). This method depends on symbolic computations. In [13] and [4] algorithms and computer programs for plotting the indefinite numerical range have been presented. These methods depend on numerical computations related with some eigenvalues and eigenvectors of $H_{\theta}$. In this section we propose an alternative algorithm that is more efficient than the existing ones in the literature, both in accuracy and in speed. We remark that our algorithm still behaves well for matrices of large size. Our approach consists in generating certain subsets of the indefinite numerical range according to Theorem 3.1, and to show that they can fill up its interior getting an accurate approximation. Since $W_{J}(T)$ is very often unbounded, this task may be somewhat difficult.

For the sake of completeness, we survey the approaches in [13] and [4]. Li-Rodman algorithm exploits the connection between the Krein numerical range of $T=\operatorname{Re}^{J} T+i \operatorname{Im}^{J} T$ and the joint numerical range of $\left(J \operatorname{Re}^{J} T, J \operatorname{Im}^{J} T, J\right)$ denoted and defined by

$$
W\left(J \operatorname{Re}^{J} T, J \operatorname{Im}^{J} T, J\right)=\left\{\left(\left\langle J \operatorname{Re}^{J} T v, v\right\rangle,\left\langle J \operatorname{Im}^{J} T v, v\right\rangle,\langle J v, v\rangle\right) \in \mathbb{R}^{3}: v \in \mathcal{H},\langle v, v\rangle=1\right\} .
$$


This connection is described by the following result (cf. [13, Proposition 1.1]).

Let $T=\operatorname{Re}^{J} T+i \operatorname{Im}^{J} T$ be an operator acting on $\mathcal{H}$. Then

$$
x+i y \in W_{J}^{+}(T) \Leftrightarrow(x, y, 1) \in \mathcal{K}\left(J \operatorname{Re}^{J} T, J \operatorname{Im}^{J} T, J\right),
$$

where $\mathcal{K}\left(J \operatorname{Re}^{J} T, J \operatorname{Im}^{J} T, J\right)$ is the convex cone generated by $W\left(J \operatorname{Re}^{J} T, J \operatorname{Im}^{J} T, J\right)$.

It is known [1] that $W\left(J \operatorname{Re}^{J} T, J \operatorname{Im}^{J} T, J\right)$ is always convex for $\operatorname{dim} \mathcal{H}>2$, and is the surface of a (possibly degenerate) ellipsoid if $\operatorname{dim} \mathcal{H}=2$. The central idea of Li-Rodman algorithm is to compute the boundary points of the compact set $W\left(J \operatorname{Re}^{J} T, J \operatorname{Im}^{J} T, J\right)$ in each direction determined by a grid point on the unit sphere in $\mathbb{R}^{3}$. Then these boundary points are joined to form a polyhedron inside $W\left(J \operatorname{Re}^{J} T, J \operatorname{Im}^{J} T, J\right)$. The points $(x / z, y / z)$, where $(x, y, z) \in W\left(J \operatorname{Re}^{J} T, J \operatorname{Im}^{J} T, J\right)$ with $z>0$, are collected and this collection of points provides an approximation for $W_{J}^{+}(T)$. Since the computations $(x / z, y / z)$ are used, and $z$ may be very small, the algorithm is not stable numerically.

The approach in [4] uses the elementary idea that the boundary of $W_{J}(T)$ may be obtained by computing the extreme eigenvalues of $\operatorname{Re}^{J}\left(\mathrm{e}^{-i \theta} T\right)$ in $\sigma_{J}^{+}\left(\mathrm{e}^{-i \theta} T\right)$ and in $\sigma_{J}^{-}\left(\mathrm{e}^{-i \theta} T\right)$ and associated $J$ unit eigenvectors $x_{\theta}^{+}$and $x_{\theta}^{-}$, for $\theta$ running over a finite mesh of points of the interval $[-\pi / 2, \pi / 2]$. The points $z_{\theta}^{+}=\left[H_{\theta} x_{\theta}^{+}, x_{\theta}^{+}\right]$and $z_{\theta}^{-}=-\left[H_{\theta} x_{\theta}^{-}, x_{\theta}^{-}\right]$are boundary points of $W_{J}^{+}(T)$ and $W_{J}^{-}(T)$, respectively [2]. As a consequence, the lines $L_{\theta}^{+}$and $L_{\theta}^{-}$with slope $\theta$ and at the distances from the origin $\lambda_{r}\left(H_{\theta}\right)$ and $\lambda_{r+1}\left(H_{\theta}\right)$, respectively, are tangents (not necessarily unique) to the boundaries of $W_{J}^{+}(T)$ and $W_{J}^{-}(T)$. Notice that these lines are supporting lines of the convex sets $W_{J}^{+}(T)$ and $W_{J}^{-}(T)$, respectively. According to this method, the boundary is approximated by a collection of points and by the line segments defined by them.

Theorem 3.1 is the key idea we use here to numerically determine $W_{J}(T)$ within some prescribed tolerance $t o l$. The respective MATLAB programs are available at the following website:

$$
\text { http://www.mat.uc.pt/ bebiano }
$$

Before we present the algorithm some considerations are in order.

Let us consider the curves $C_{1}^{+}, C_{2}^{+}, \ldots, C_{r}^{+}\left(C_{1}^{-}, C_{2}^{-}, \ldots, C_{s}^{-}\right)$generated, as described in Theorem 3.1, by vectors with positive norm (negative norm). Let $K^{+}=\operatorname{conv}\left(C_{1}^{+}, C_{2}^{+}, \ldots, C_{r}^{+}\right), K^{-}=$ $\operatorname{conv}\left(C_{1}^{-}, C_{2}^{-}, \ldots, C_{s}^{-}\right)$. The pseudo-convex hull of $C_{1}^{+}, C_{2}^{+}, \ldots, C_{r}^{+}, C_{1}^{-}, C_{2}^{-}, \ldots, C_{s}^{-}$, denoted $\operatorname{pconv}\left(C_{1}^{+}\right.$, $\left.C_{2}^{+}, \ldots, C_{r}^{+}, C_{1}^{-}, C_{2}^{-}, \ldots, C_{s}^{-}\right)$, is the union of all half-rays of the lines passing through $z^{+} \in K^{+}$, $z^{-} \in K^{-}$with endpoint in $z^{+}$not containing $z^{-}$, or with endpoint in $z^{-}$not containing $z^{+}$.

Suppose $T \in M_{n}$ and a pre-specified level of tolerance tol are given. The tol depends on the machine precision and how much of the unbounded region one wants to generate. When we are dealing with nondegenerate numerical ranges, we are interested in finding an interval $\left[\theta_{\min }, \theta_{\max }\right], 0<\theta_{\max }-\theta_{\min } \leq \pi$, 
such that for $\theta$ in that interval the conditions (i), (ii) and (iii) of Section 2 are fulfilled. For commodity, such a $\theta$ will be called an admissible angle. Contrarily, $\theta$ is said to be non-admissible.

If we wish to computationally generate the numerical range of an arbitrary matrix $T=\operatorname{Re}^{J} T+$ $i \operatorname{Im}^{J} T$, our first task is to test whether the matrix belongs to the class $\mathcal{N} \mathcal{D}$. As a preliminary test we should check whether 0 is in the corresponding joint numerical range $W\left(J \operatorname{Re}^{J} T, J \operatorname{Im}^{J} T, J\right)$. If this is the case, $W_{J}(T)$ is degenerate (cf. [13, Proposition 2.4]) and $T \notin \mathcal{N D}$. Indeed, $T \in \mathcal{N D}$ if and only if it is not a scalar matrix and $0 \notin W\left(J \operatorname{Re}^{J} T, J \operatorname{Im}{ }^{J} T, J\right)$. If $T \in \mathcal{N} \mathcal{D}$, we have to search for an interval $\left[\theta_{\text {min }}, \theta_{\text {max }}\right], 0<\theta_{\text {max }}-\theta_{\text {min }}<\pi$ of admissible angles $\theta$. For a real matrix in $\mathcal{N D}, \theta=0$ is an admissible angle while $\theta= \pm \pi / 2$ are non-admissible angles. In general, after a convenient rotation, for any complex matrix in the class $\mathcal{N} \mathcal{D}, \theta=0$ is an admissible angle, while $\theta= \pm \pi / 2$ are non-admissible angles. So we shall restrict our attention to this case.

\subsection{Algorithm}

Step 0. Search for an admissible angle $\theta$. If the matrix is complex, we test the angle $-\pi / 2$ for this property. If the answer is positive, go to Step 1.

If not, bisect the interval $[-\pi / 2, \pi / 2]$. If $\theta=0$ is admissible, we proceed to Step 1 .

Otherwise, we continue analyzing the angles in the sets

$$
\{-p i / 4, \pi / 4\},\{-3 \pi / 8,-\pi / 8, \pi / 8,3 \pi / 8\},\{-5 \pi / 16,-3 \pi / 16,-\pi / 16,3 \pi / 16,5 \pi / 16\}, \ldots
$$

until, for some $k$, one of the angles $\theta_{\ell, k}=-2^{k-1} \pi / 2^{k}+(2 \ell-1) \pi / 2^{k}, \ell=0,1, \ldots, 2^{k-1}$, in the set

$$
\left\{\frac{\left(1-2^{k-1}\right) \pi}{2^{k}}, \frac{\left(3-2^{k-1}\right) \pi}{2^{k}}, \frac{\left(5-2^{k-1}\right) \pi}{2^{k}}, \ldots, \frac{\left(2^{k-1}-1\right) \pi}{2^{k}}\right\}
$$

is admissible, and we proceed to Step 1.

Replacing the matrix $T$ by $\mathrm{e}^{-i \theta_{\ell, k}} T$, where $\theta_{\ell, k}$ is admissible for $T$, then $\theta=0$ is admissible for the rotated matrix.

Step 1. Choice of $\left[\theta_{\min }, \theta_{\text {max }}\right]$ Fix a tolerance $t o l=\pi / 2^{N}, N \geq 4$. Suppose $\theta=0$ is an admissible angle. Construct a set of admissible angles, starting with $\theta_{0}=0$, as follows. Bisect successively the interval $[0, \pi / 2]$. until we find an admissible angle $\theta_{1}=\pi / 2^{\nu_{1}}$, the integer $\nu_{1}$ being such that the angle $\theta_{1}+\pi / 2^{\nu_{1}}$ is non-admissible. Proceed in this way until we find a new admissible angle $\theta_{2}=$ $\pi / 2^{\nu_{1}}+\pi / 2^{\nu_{1}+\nu_{2}}$, the integer $\nu_{2}$ being such that the angle $\theta_{2}+\pi / 2^{\nu_{1}+\nu_{2}}$ is non-admissible, and so on, until we reach the angle $\theta_{k}=\pi / 2^{\nu_{1}}+\pi / 2^{\nu_{1}+\nu_{2}}+\cdots+\pi / 2^{\nu_{1}+\nu_{2}+\ldots+\nu_{k}}$, which is admissible, while the angle $\theta_{k}+\pi / 2^{\nu_{1}+\nu_{2}+\cdots+\nu_{k}}$ is non-admissible, being $\nu_{1}+\nu_{2}+\cdots+\nu_{k} \leq N$. Similarly, we obtain the admissible angles $\bar{\theta}_{1}=-\pi / 2^{\bar{\nu}_{1}}, \bar{\theta}_{2}=-\pi / 2^{\bar{\nu}_{1}}-\pi / 2^{\bar{\nu}_{1}+\bar{\nu}_{2}}, \ldots, \bar{\theta}_{\ell}=-\pi / 2^{\bar{\nu}_{1}}-\pi / 2^{\bar{\nu}_{1}+\bar{\nu}_{2}}-$ 
$\cdots-\pi / 2^{\bar{\nu}_{1}+\bar{\nu}_{2}+\cdots+\bar{\nu}_{\ell}}$. If the matrix is real, it is obvious that $\bar{\theta}_{j}=-\theta_{j}, j=1, \cdots k$. The required interval of admissible angles is $\left[\theta_{\min }, \theta_{\max }\right]=\left[\bar{\theta}_{\ell}, \theta_{k}\right]$, and continue.

Step 2. Set $\theta_{k}=\theta_{\text {min }}+(k-1)\left(\theta_{\max }-\theta_{\text {min }}\right) / m, k=1, \ldots, m+1$ for some positive integer $m \geq 3$. For each $\theta_{k}$, construct the $J$-Hermitian matrix $\operatorname{Re}^{J}\left(\mathrm{e}^{-i \theta_{k}} T\right)$ and compute its eigenvalues.

Step 3. Starting with $k=1$, up to $k=m+1$, take the following steps:

(i) Compute eigenvectors $u_{k}$ and $v_{k}$ associated, respectively, to the largest eigenvalue in $\sigma_{J}^{-}\left(\operatorname{Re}^{J}\left(\mathrm{e}^{-i \theta_{k}} T\right)\right)$ and to the smallest eigenvalue in $\sigma_{J}^{+}\left(\operatorname{Re}^{J}\left(\mathrm{e}^{-i \theta_{k}} T\right)\right)$.

(ii) Compute the $J$-compression of $T$ to the subspace $\operatorname{span}\left\{u_{k}, v_{k}\right\}, T_{\tilde{u}_{k}} \tilde{v}_{k}$.

(iii) Compute the boundary of $W_{J_{\tilde{u}_{k}} \tilde{v}_{k}}\left(T_{\tilde{u}_{k} \tilde{v}_{k}}\right), \Gamma_{k}$.

(iv) If $k<m+1$, take the next $k$ value and return to (i). Otherwise, continue.

Step 4. Plot, separately, the convex-hulls of the positive and of the negative branches of the collection of hyperbolas $\Gamma_{1}, \ldots, \Gamma_{m}$, taking care, for each hyperbola, which branch is in $W_{J}^{+}(T)$ and in $W_{J}^{-}(T)$. Then take their pseudo convex hull, as an approximation for $W_{J}(T)$. If there are common tangents to the boundaries of both convex-hulls, then $\partial W_{J}(T)$ will have flat portions at infinity.

This algorithm may not be efficient when the numerical ranges of the compressed matrices degenerate into line segments or half-rays. This is the case when $T$ is a direct sum of blocks. This suggests a modified algorithm in which the choice of generating vectors for boundary points is more convenient.

The Steps from $0^{\prime}$ to $2^{\prime}$ are as in the previous algorithm.

Step $3^{\prime}$. Compute eigenvectors $u_{1}$ and $v_{1}$ associated, respectively, to the largest eigenvalue in $\sigma_{J}^{-}\left(\operatorname{Re}^{J}\left(\mathrm{e}^{-i \theta_{1}} T\right)\right)$ and to the smallest eigenvalue in $\sigma_{J}^{+}\left(\operatorname{Re}^{J}\left(\mathrm{e}^{-i \theta_{1}} T\right)\right)$.

Step $4^{\prime}$. Starting with $k=2$ and up to $k=m+1$, take the following steps:

(i) Compute eigenvectors $u_{k}$ and $v_{k}$ associated, respectively, to the largest eigenvalue in $\sigma_{J}^{-}\left(\operatorname{Re}^{J}\left(\mathrm{e}^{-i \theta_{k}} T\right)\right)$ and to the smallest eigenvalue in $\sigma_{J}^{+}\left(\operatorname{Re}^{J}\left(\mathrm{e}^{-i \theta_{k}} T\right)\right)$.

(ii) Compute the $J$-compressions of $T$ to the subspaces $\operatorname{span}\left\{u_{k}, u_{k-1}\right\}$ and $\operatorname{span}\left\{v_{k}, v_{k-1}\right\}$, respectively $T_{\tilde{u}_{k}} \tilde{u}_{k-1}$ and $T_{\tilde{v}_{k}} \tilde{v}_{k-1}$.

(iii) Compute and draw the boundaries of $W_{J_{\tilde{u}_{k}} \tilde{u}_{k-1}}\left(T_{\tilde{u}_{k} \tilde{u}_{k-1}}\right)$ and of $W_{J_{\tilde{v}_{k}} \tilde{v}_{k-1}}\left(T_{\tilde{v}_{k} \tilde{v}_{k-1}}\right)$, respectively $\Gamma_{k}$ and $\Lambda_{k}$.

(iv) If $k<m+1$, take next $k$ value and return to (i). Otherwise, continue. 
Step $5^{\prime}$. Take the following steps.

(i) Compute the $J$-compressions of $T$ to the subspaces $\operatorname{span}\left\{u_{1}, v_{1}\right\}$ and $\operatorname{span}\left\{v_{m+1}, u_{m+1}\right\}$, respectively $T_{\tilde{u}_{1} \tilde{v}_{1}}$ and $T_{\tilde{v}_{m+1} \tilde{u}_{m+1}}$.

(ii) Compute the boundaries of $W_{J_{\tilde{v}_{1} \tilde{u}_{1}}}\left(T_{\tilde{v}_{1} \tilde{u}_{1}}\right)$ and $W_{\tilde{\tilde{u}}_{m+1} \tilde{v}_{m+1}}\left(T_{\tilde{u}_{m+1} \tilde{v}_{m+1}}\right)$, respectively $\Gamma_{m+2}$ and $\Lambda_{m+2}$.

Step $6^{\prime}$. Take the convex-hulls of the positive and the negative branches of the collection of conics $\Gamma_{1}, \Gamma_{2}, \ldots, \Gamma_{m+2}, \Lambda_{1}, \Lambda_{2}, \ldots, \Lambda_{m+2}$. Then take their pseudo-convex hull as an approximation for $W_{J}^{ \pm}(T)$. If there are common tangents to the boundaries of both convex-hulls, then $\partial W_{J}(T)$ will have flat portions at infinity.

Remark 4.1 If a flat portion exists in the boundary of $W_{J}(T)$, then $\operatorname{Re}^{J}\left(\mathrm{e}^{-i \theta} T\right)$ has multiple eigenvalues for some $\theta$. If such a direction is found, then the associated flat portion may be easily produced. For instance, suppose that $\operatorname{Re}^{J} T$ has a multiple eigenvalue in the situation of Step $4(i)$. Let $u_{a}, u_{b}$ be linearly independent eigenvectors associated with it. Take $u(\alpha)=u_{a}+\alpha u_{b}$ with $\alpha$ real and compute the extreme values of $\left[\operatorname{Im}^{J} T u(\alpha), u(\alpha)\right] /[u(\alpha), u(\alpha)]$. The flat portion is so produced.

Remark 4.2 If there is a corner in $\partial W_{J}^{+}(T)$ (or $\partial W_{J}^{-}(T)$ ), then it may happen that the vectors $u_{k}, u_{k+1}, \ldots, u_{k+l}$ are pairwise linearly dependent. If that happens, the conics associated with the (onedimensional) spaces $\operatorname{span}\left\{u_{k}, u_{k+1}\right\}, \ldots, \operatorname{span}\left\{u_{k+l-1}, u_{k+l}\right\}$ (or $\left.\operatorname{span}\left\{v_{k}, v_{k+1}\right\}, \ldots, \operatorname{span}\left\{v_{k+l-1}, v_{k+l}\right\}\right)$ degenerate trivially into the point $\left[u_{k}, u_{k}\right]\left(\left[v_{k}, v_{k}\right]\right)$, which is easily found.

\section{Discussion and examples}

We have judiciously chosen optimal compressions, instead of considering randomly generated compression vectors. We observe that, in general, the modified algorithm of Section 4 provides a much better accuracy than the preliminary algorithm in the approximation of $W_{J}(T)$. Remarkably, both behave especially well when compared with the one in [4], which merely provides a polygonal approximation of $W_{J}(T)$, and so it requires a much bigger mesh to reach a convenient accuracy. The algorithm of Section 4.1 provides branches of hyperbolas whose convex-hull should be determined. Henceforth, small flat portions may arise when joining consecutive branches of hyperbolas. In the modified algorithm, the interpolation between consecutive boundary points is made by arcs of ellipses, so spurious flat portions do not in general arise. In the event the boundary of $W_{J}(T)$ has "flat" portions but it is not polygonal, the algorithms work well (cf. Examples 5.2 and 5.3), and are also efficient in the extreme case of a polygonal boundary. We recall that the computational cost for determining $W_{J}(T)$ 
by approximating its boundary curve $\partial W_{J}(T)$ by eigenvalues and eigenvectors evaluations requires $\mathcal{O}\left(n^{3}\right)$ operations per point, while finding 2 by 2 compressions for $T$ is an $\mathcal{O}\left(n^{2}\right)$ process. Finally we notice that the algorithms apply on the definite case $J=I$.

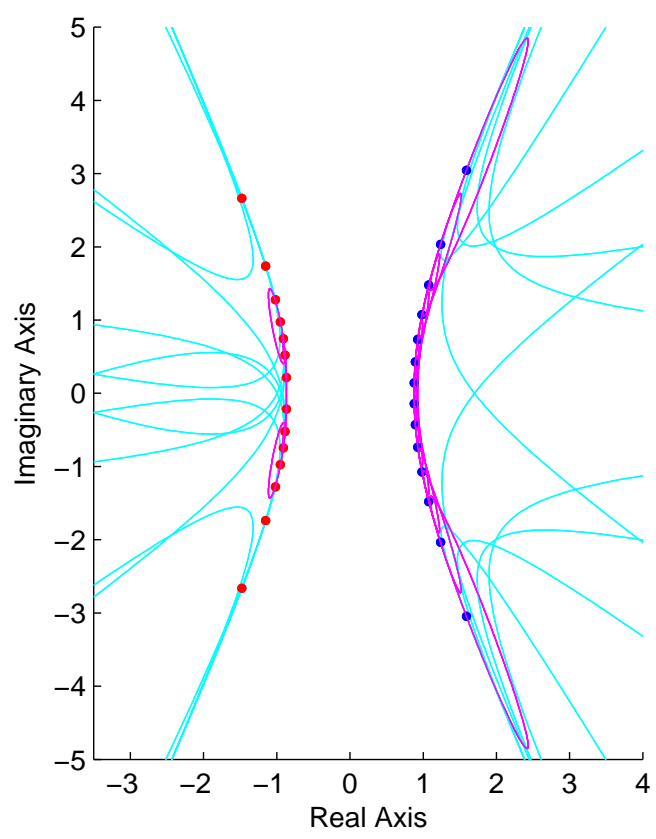

Figure 1: $W_{J}(T)$ for Example 5.1

Example 5.1 We illustrate in Figure 1, the indefinite numerical range of a pentadiagonal matrix of order 50 with main diagonal $-1,2,-1,2, \ldots$, first super diagonal $1,-1,1,-1, \ldots$, and first and second subdiagonals $1,0,1,0, \ldots$ and $0,1,0,1, \ldots$, respectively. We considered $m=15$ and took $\theta_{\max }=$ $-\theta_{\min }=0.4172$. The computation was done with MATLAB R2012b on a OpenSUSE Linux 12.2 computer equiped with Intel(R) Xeon(R) E5520 (2.26 GHz, quad core) and 48 GB of RAM.

To compare the accuracy of numerical ranges plotters we use the idea in [18], via their enclosed partial areas, namely, the areas determined by a line through the points $x=-6$ and $x=6$ and $\partial W_{J}(T)$. When comparing the effort to achieve several accurate leading digits for the searched partial areas, we may conclude that our 2 by 2 compressions matrix algorithm is faster and more accurate than the algorithms in [4] and [13]. As the table shows, our algorithm achieves a more quickly stabilization than the others. 


\begin{tabular}{|c|c|c|c|c|}
\hline Algorithm & $m$ & Seconds & Eigenanalyses & Area \\
\hline \hline Present algorithm & 7 & 0.883730 & 24 & 149.2469 \\
& 14 & 1.062783 & 31 & 149.3682 \\
& 28 & 1.255899 & 45 & 149.4168 \\
\hline Algorithm from [13] & 7 & 1.204616 & 812 & 147.7302 \\
& 14 & 3.531369 & 3192 & 149.0175 \\
& 28 & 14.676580 & 12656 & 149.3215 \\
\hline Algorithm from [4] & 7 & 0.941884 & 42 & 133.2116 \\
& 14 & 2.356972 & 84 & 136.6461 \\
& 28 & 8.335534 & 168 & 142.5310 \\
\hline
\end{tabular}

Table 1: Performance of algorithms from [14], [4] and the present one.

Example 5.2 Figure 2 refers to $W_{J}(T)$, with

$$
T=\left[\begin{array}{ccc}
i & 0 & \frac{1}{2} \\
0 & 0 & \frac{1}{2} \\
\frac{1}{2} & \frac{1}{2} & -\sqrt{2}
\end{array}\right], \quad J=\operatorname{diag}(1,1,-1),
$$

being $m=3, \theta_{\min }=-0.5400, \theta_{\max }=0.8345$,

Example 5.3 Figure 3 refers to $W_{J}(T)$, with

$$
T=\left[\begin{array}{cccccc}
2+i & 0 & -1 & 0 & 0 & 0 \\
0 & -2 & 0 & -1 & 0 & 0 \\
2 & 0 & 2+i & 0 & -1 & 0 \\
0 & 2 & 0 & -2 & 0 & -1 \\
0 & 0 & 2 & 0 & 2+i & 0 \\
0 & 0 & 0 & 2 & 0 & -2
\end{array}\right], \quad J=\operatorname{diag}(1,-1,1,-1,1,-1)
$$

being $m=3, \theta_{\max }=0.8647, \theta_{\min }=-0.6122$. The tolerance in Step 1 of the algorithm was fixed taking $N=15$. Notice that there exists a permutation matrix $P$ such that $T=P\left(T_{1} \oplus T_{2}\right) P^{-1}, J=$ $P\left(I_{3} \oplus-I_{3}\right) P^{-1}$ and that $\theta_{\max }, \theta_{\text {min }}$ give the directions of the flat portions extending to infinity.

\section{Concluding remarks}

We have approximated Krein space numerical ranges by compression methods, in particular we have developed the Marcus-Pesce process [16]. To this end, we have judiciously generated 2 by 2 


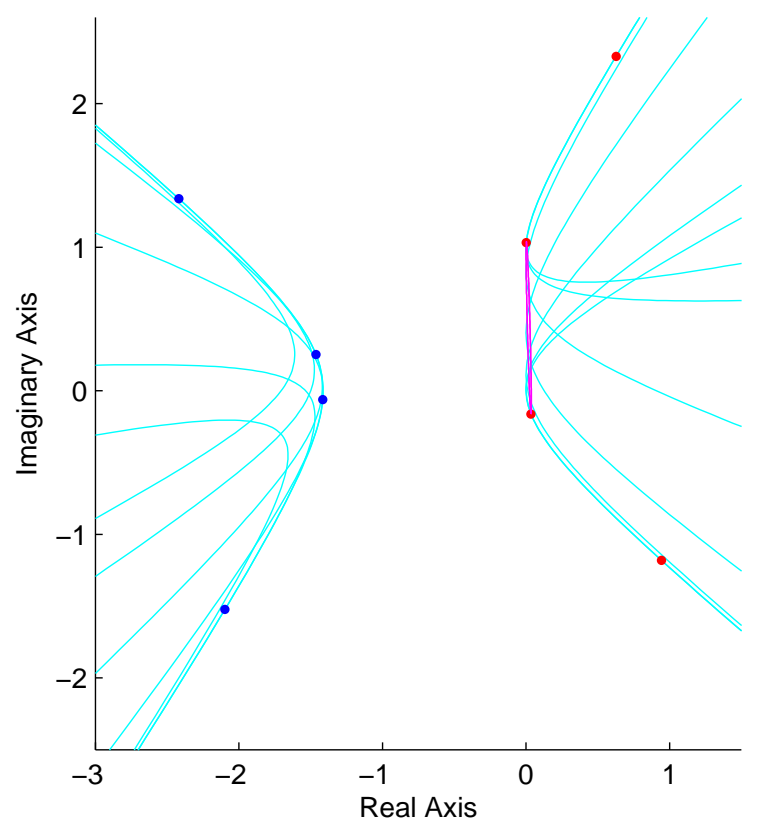

Figure 2: $W_{J}(T)$ for Example 5.2

matrix compressions, and their easily determined elliptical and hyperbolical numerical ranges. Our approach is essentially the standard one for Hilbert space numerical ranges [18], except that here anisotropic vectors (i.e, vectors with vanishing norm) can occur, and the inner product defined by the identity matrix $I_{n}$ in Hilbert spaces becomes now indefinite, and defined by the involution $J$. Pairs of vectors $u$ and $v$ with $u^{*} J u \times v^{*} J v>0$ behave as in the definite case providing elliptical numerical ranges, while those with $u^{*} J u \times v^{*} J v<0$ originate hyperbolical numerical ranges for the boundary curve approximations.

We emphasize that the presented algorithm plays a crucial role in obtaining solution vectors for the inverse indefinite numerical range problem, namely in the case of large dimension matrices and given points near to the boundary (see [7]).

\section{References}

[1] Y.H. Au-Yeung and N.K. Tsing, An extension of the Hausdorff-Toeplitz theorem on the numerical range, Proc. Amer. Math. Soc., 89 (1983) 215-218. 


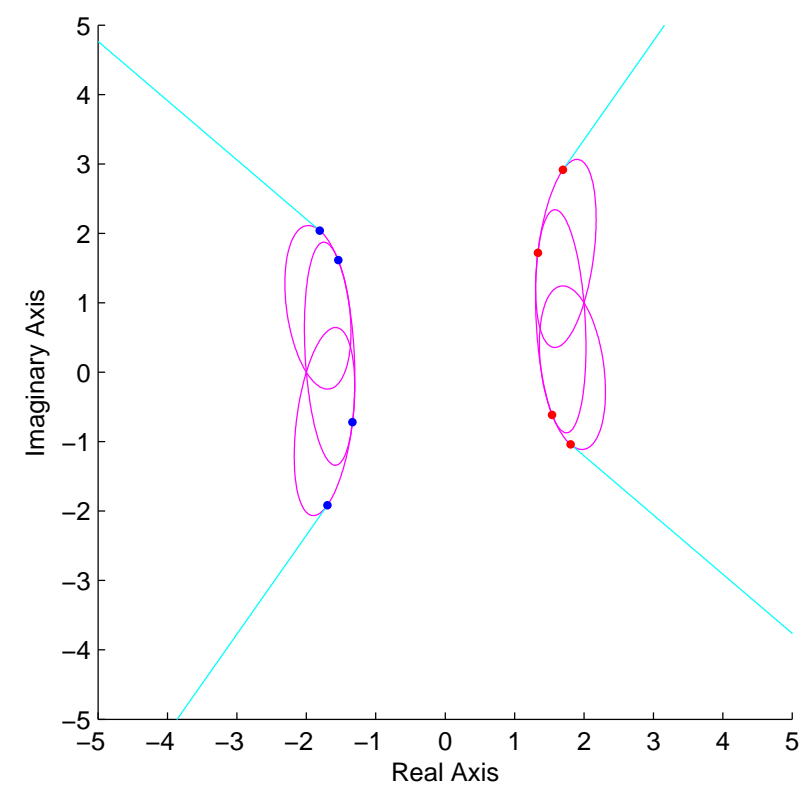

Figure 3: $W_{J}(T)$ for Example 5.3

[2] N. Bebiano, R. Lemos, J. da Providência and G. Soares, On generalized numerical ranges of operators on an indefinite inner product space, Linear Multilin. Algebra 52 No. 3-4, (2004) 203-233.

[3] N. Bebiano, H. Nakazato, J. da Providência, R. Lemos and G. Soares, Inequalities for J-Hermitian matrices, Linear Algebra Appl. 407 (2005) 125-139.

[4] N. Bebiano, J. da Providência, A. Nata and G. Soares, Krein Spaces Numerical Ranges and their Computer Generation, Electron. J. Linear Algebra, 17 (2008) 192-208.

[5] N. Bebiano, J. da Providência, R. Teixeira, Flat portions on the boundary of the indefinite numerical range of $3 \times 3$ matrices, Linear Algebra Appl. 428 (2008) 2863-2879.

[6] N. Bebiano, I. Spitkovsky, Numerical ranges of Toeplitz operators with matrix symbols, Linear Algebra Appl. 436 (2012) 1721-1726.

[7] N. Bebiano, J. da Providência, A. Nata and J. P. da Providência, An inverse problem for the indefinite numerical range, $C M U C$ preprint. . 
[8] M.-T. Chien and H. Nakazato, The numerical range of a tridiagonal operator, J. Math. Anal. Appl., 373, No. 1 (2011), 297-304.

[9] C. Crorianopoulos, P. Psarrakos and F. Uhlig. A method for the inverse numerical range problem. Linear Algebra its Appl. 24 (2010) 055019.

[10] I.Gohberg, P.Lancaster and L.Rodman, Matrices and Indefinite Scalar Product. Birkhäuser, Basel-Boston, 1983.

[11] R.A. Horn and C.R. Johnson, Matrix Analysis. Cambridge University Press, New York, 1985.

[12] R.A. Horn and C.R. Johnson, Topics in Matrix Analysis. Cambridge University Press, Cambridge, 1991.

[13] C.-K. Li and L. Rodman, Shapes and computer generation of numerical ranges of Krein space operators. Electron. J. Linear Algebra, 3 (1998) 31-47.

[14] C.-K. Li and L. Rodman, Remarks on numerical ranges of operators in spaces with an indefinite metric, Proc. Amer. Math. Soc. 126 No. 4, (1998) 973-982.

[15] C.-K. Li, N.K. Tsing and F. Uhlig. Numerical ranges of an operator on an indefinite inner product space. Electron. J. Linear Algebra 1 (1996) 1-17.

[16] M. Marcus and C. Pesce, Computer generated numerical ranges and some resulting theorems. Linear Multilinear Algebra, 20 (1987), 121-157.

[17] P.J. Psarrakos, Numerical range of linear pencils, Linear Algebra Appl. 317 (2000) 127-141.

[18] F. Uhlig, Faster and more accurate computation of the field of values for $\mathrm{n}$ by $\mathrm{n}$ matrices, Linear and Multilinear Algebra (2013) DOI: 10.1080/03081087.2013.779269. 